\title{
DETERMINACIÓN DE MACRO Y MICRONUTRIENTES CATIÓNICOS EN TEJIDOS VEGETALES DE BANANO, PALMA Y ROSAS, POR ESPECTROSCOPÍA DE EMISIÓN POR ACOPLAMIENTO DE PLASMA INDUCTIVO Y DETECCIÓN ÓPTICA (ICP-OES)
}

DETERMINATION OF MACRO AND MICRO CATIONIC NUTRIENTS IN BANANA, PALM AND ROSES PLANT TISSUES, USING INDUCTIVELY COUPLED PLASMA OPTICAL EMISSION SPECTROMETRY (ICP-OES)

Luis Cacuango P. ${ }^{1}$, Soraya Alvarado O. ${ }^{2}$ \& Yolanda Jibaja A. ${ }^{1}$

Palabras clave: nutrientes, tejidos vegetales, espectroscopia, ICP-OES, AA. Keywords: nutrients, plant tissues, spectroscopy, ICP-OES, AA.

\section{RESUMEN}

Se determinó la concentración de macro y micronutrientes catiónicos: $\mathrm{K}$, Ca, $\mathrm{Mg}$, Zn, Cu. Fe y Mn, en muestras de tejido vegetal de banano, palma y rosas, por espectroscopia de emisión por acoplamiento de plasma inductivo y detec-

1 Pontificia Universidad Católica del Ecuador, Facultad de Ciencias Exactas y Naturales, Escuela de Ciencias Químicas, Quito Ecuador (Ihcacuango@gmail.com; yjibaja@puce.edu.ec).

2 Instituto Nacional Autónomo de Investigaciones Agropecuarias (INIAP), Departamento de Manejo de Suelos y Aguas (DMSA), Quito Ecuador (spalv_2000@yahoo.com). 
ción óptica (ICP-OES). La parte experimental se desarrolló en los laboratorios del Departamento de Manejo de Suelos y Aguas (DMSA) de la Estación Experimental Santa Catalina del INIAP, los cuales utilizan la técnica de Absorción Atómica para el análisis de elementos en muestras foliares; dicha técnica presenta limitaciones en cuanto a límites de detección, efectos de matriz, rango lineal y número de elementos que pueden ser analizados en forma simultánea. En cambio, la espectroscopia de emisión con plasma acoplado inductivamente, es una técnica que está a la vanguardia como herramienta analítica para la determinación multielemental en tejidos foliares. Por esta razón se optimizó la técnica de ICP-OES, para el análisis de macro y micronutrientes catiónicos en muestras de tejido vegetal, ya que con el uso de este equipo se incrementará la capacidad operativa del laboratorio. Los análisis se realizaron con muestras de tejido vegetal de banano, palma y rosas, que se utilizan como material de referencia para los análisis de rutina de muestras vegetales que se realizan en los laboratorios del DMSA; se utilizó como Material de Referencia Certificado (MRC) el Standard Reference Material 1515, Apple Leaves. Las muestras y el material certificado se sometieron a digestión húmeda para luego cuantificar los analitos en estudio por medio de las técnicas de AA e ICP-OES. Se realizó un estudio comparativo de las variables: exactitud, precisión, linealidad, límites de detección y cuantificación en el cual se determinó que existen diferencias significativas entre las técnicas empleadas. El análisis estadístico demostró que existen diferencias altamente significativas en el contenido de macro y micronutrientes en los tejidos analizados, como sucede en el caso del magnesio en tejido foliar de palma, cuyos porcentajes fueron de 0,36 y $0,52 \%$, obtenidos mediante AA e ICP respectivamente y dentro del grupo de los micronutrientes, el contenido de hierro en tejido foliar de palma con valores de 182,48 y 122,33 $\mathrm{mg} / \mathrm{L}$. Sin embargo, en el caso de las muestras de tejido foliar de banano, palma y rosas, los valores obtenidos mediante AA e ICP, en el análisis de potasio, demostraron que estadísticamente la diferencia es significativa.

\section{ABSTRACT}

This study is focus on determine the concentration of macro and micro cationic nutrients: $\mathrm{K}, \mathrm{Ca}, \mathrm{Mg}$, Zn, Cu, Fe and Mn in plant tissue samples banana, palm, 
roses, by inductively coupled plasma optical emission spectrometry (ICP-OES). The experimental part of this research was developed in the laboratories of the Department of Soil and Water Management (DMSA) in the Santa Catalina Experimental Station of INIAP, which use atomic absorption technique for the analysis of elements in leaf samples; this technique has limitations in terms of limits of detection, matrix effects, linear range, and number of elements that can be analyzed simultaneously. However, emission spectroscopy with inductively coupled plasma is a technique that is at the forefront as an analytical tool for multielement determination in leaf tissues. For this reason the technique ICP-OES is optimized, for analysis of macro and micro cationic nutrients in samples of plant tissue, since the use of this equipment will increase the operational capacity of the laboratory. Analyses were carried out on samples of plant tissue of banana, palm and roses, which are used as reference material in routine analysis of plant samples carried out in DMSA laboratories; Apple Leaves 1515 was used as a Certified Reference Material. Samples and standard reference material subjected to wet digestion and then quantify the analytes under study through the techniques of AA and ICP-OES. A comparative study was performed of the variables: accuracy, precision, linearity, limits of detection and quantification, in which it was determined there are significant differences between the techniques used. Statistical analysis showed that there were highly significant differences in the content of macro and micronutrients in the tissues analyzed. In the case of magnesium in palm leaf tissue, the percentages were 0.36 and $0.52 \%$, obtained by AA and ICP respectively, and within the group of micronutrients, iron content in palm leaf tissue with values of 182.48 and $122.33 \mathrm{mg} / \mathrm{L}$. However, in the case of the samples of leaf tissue of banana, palm and roses, the values obtained by ICP-OES and AA in potassium analysis, showed that the difference is statistically significant. 


\section{INTRODUCCIÓN}

El análisis de tejido vegetal se refiere al análisis químico cuantitativo de los nutrientes esenciales en los tejidos de las plantas. Por varios años, este tipo de análisis se realizó para determinar el estado nutricional de los cultivos arbustivos debido a su naturaleza y al extenso sistema radicular que poseen; sin embargo, el análisis de tejido vegetal ha adquirido mayor importancia a medida que se desarrolla el conocimiento acerca de la nutrición de las plantas, de los requerimientos de nutrientes durante todo el ciclo del cultivo, y la posibilidad de aplicarlos mediante modernos sistemas de riego.

La parte foliar de las plantas es la más utilizada para realizar el análisis de tejido vegetal ya que es el órgano principal donde se elaboran las sustancias nutritivas y refleja el estado nutricional (ICA, 2012). Varias investigaciones han documentado una alta correlación entre el contenido de nutrientes a nivel foliar y el desarrollo y producción del cultivo (Ulrich, 1972).

Desde hace décadas se ha utilizado la técnica de absorción atómica de llama para la determinación de elementos en muestras foliares. Afectan a la técnica, en distintos grados, el tipo de matriz, el rango de concentración de los elementos a analizar, además, los digestados de las muestras requieren usualmente un tratamiento adecuado para llevar la concentración de los analitos al rango analítico adecuado para el análisis; por estas razones, la técnica se vuelve lenta y problemática, comparada con técnicas instrumentales multielementales como la espectroscopia de emisión acoplada inductivamente a plasma (Briceño \& Pacheco, 1984).

La espectroscopia de emisión por acoplamiento de plasma se ha vuelto una técnica ampliamente empleada para el análisis de soluciones, con inclusión de los digestados de tejidos vegetales, está relativamente libre de los efectos de matriz y tiene una excelente sensibilidad en concentraciones muy bajas (partes por billón) con un rango de concentración utilizable de cuatro a cinco cifras (Alvarado, Yglesias, \& Güel, 2005). 
Otra de las ventajas que presenta la espectroscopia de emisión acoplada inductivamente a plasma, ICP-OES, se debe a las altas temperaturas que alcanza el equipo, alrededor de $10000 \mathrm{~K}$, a esa temperatura se reducen o eliminan muchas interferencias químicas que se pueden presentar en absorción atómica, estas temperaturas permiten mejorar la eficiencia de la excitación y la ionización de las partículas, lo que ayuda a reducir los límites de detección (Boss \& Fredeen, 1997).
El objetivo de este trabajo es comparar el contenido de macro y micronutrientes en tejido foliar de banano, palma y rosas, cultivos económicamente importantes para el Ecuador, analizados mediante las técnicas de Absorción Atómica de Ilama (AA) y espectroscopia de emisión acoplada inductivamente a plasma (ICP-OES, por sus siglas en inglés).

\section{MATERIALES Y MÉTODOS}

\section{Muestras}

Las muestras de tejido vegetal de banano, palma y rosas, empleadas en el análisis, fueron suministradas por el Departamento de Manejo de Suelos y Aguas (DMSA) del INIAP. Dichas muestras son usadas como material de referencia y se las emplea en el análisis de rutina de tejidos foliares del laboratorio.

La preparación de estas muestras consistió en el lavado con agua destilada con el fin de eliminar cualquier tipo de contaminación: polvo, suelo, otros tejidos, fertilizantes, fungicidas, etc. Luego se secaron a $60{ }^{\circ} \mathrm{C}$ durante 48 horas aproximadamente, se molieron y tamizaron con una malla de 40 mesh.

Las muestras y el material de referencia son almacenados a temperatura ambiente hasta el momento del análisis.

\section{Extracción de los analitos}

Las muestras de tejido vegetal se sometieron a digestión húmeda con ácido nítrico grado analítico al 65\% 
y ácido perclórico grado analítico al $72 \%$ en relación $5: 1$, hasta obtener una solución transparente. Una vez digeridas se dejaron enfriar a temperatura ambiente y se aforaron a $25 \mathrm{~mL}$ con agua destilada tipo I. Los extractos obtenidos se leyeron en el espectrofotómetro de Absorción Atómica modelo AA-680 (Shimadzu), y en el espectrofotómetro ICP-OES modelo Optima 5300 DV (Perkin Elmer).

\section{Análisis estadístico}

Se realizó el análisis estadístico (ANOVA) para determinar si hay diferencias significativas entre los resultados obtenidos por espectrofotome- tría AA y espectrofotometría ICP.

\section{RESULTADOS}

Las condiciones óptimas de operación del espectrofotómetro de emisión por acoplamiento de plasma inducido para la determinación de macro y micronutrientes en tejido vegetal, son las indicadas en la Tabla 1.

Tabla 1. Condiciones de operación del equipo ICP-OES OPTIMA 5300 DV

\begin{tabular}{lll}
\hline Elemento & $\begin{array}{c}\text { Longitud } \\
\text { de onda }\end{array}$ & $\begin{array}{c}\text { Posición de } \\
\text { la llama }\end{array}$ \\
\hline $\mathrm{K}$ & 766,490 & Radial \\
\hline $\mathrm{Ca}$ & 315,887 & Radial \\
\hline $\mathrm{Mg}$ & 279,077 & Radial \\
\hline $\mathrm{Zn}$ & 213,857 & Axial \\
\hline $\mathrm{Cu}$ & 324,752 & Axial \\
\hline $\mathrm{Fe}$ & 259,939 & Axial \\
\hline $\mathrm{Mn}$ & 257,610 & Axial \\
\hline
\end{tabular}

Luego de la evaluación del método se determinaron los macro y micronutrientes catiónicos en las muestras de tejidos vegetales de banano, palma y rosas.

\section{Potasio}

En la Figura 1, se presentan las concentraciones de potasio en tejido vegetal de banano, palma y rosas.

El contenido de este elemento en tejido foliar de banano es más alto respecto al de las muestras de palma y rosas, y estadísticamente la diferencia es altamente significativa; si se comparan las técnicas de análisis, los resultados obtenidos mediante ICP-OES 


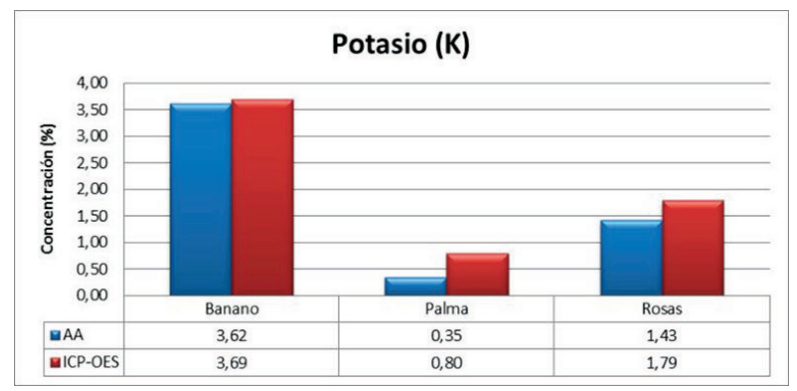

Figura 1. Potasio en muestras de tejido vegetal

son ligeramente mayores a los obtenidos por AA, una mayor diferencia se observa en las muestras de palma, cuyo contenido de potasio es de 0,80 y $0,35 \%$ respectivamente; sin embargo, al comparar las técnicas empleadas en cada tejido analizado, estadísticamente no existen diferen- cias significativas entre las técnicas empleadas en el análisis.

\section{Calcio}

En la Figura 2 se presentan las concentraciones de calcio en tejido vegetal de banano, palma y rosas.

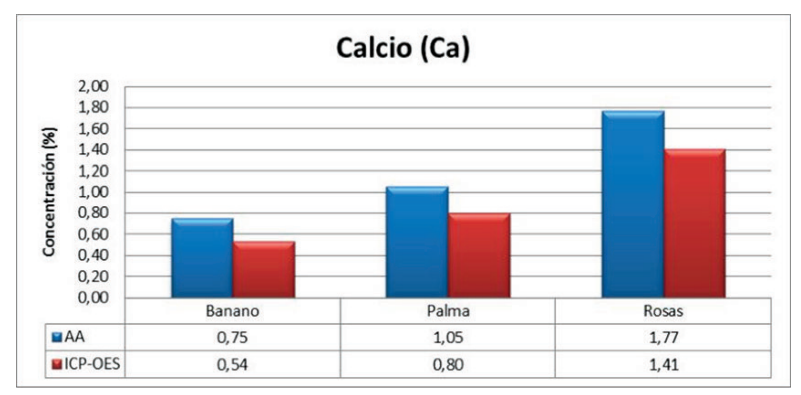

Figura 2. Calcio en muestras de tejido vegetal

El tejido vegetal de rosas presenta el contenido de calcio más alto, respecto a las muestra de tejido vegetal de banano y palma, así lo demuestran los resultados obtenidos con las dos técnica empleadas en el análisis, 
así mismo, en esta muestra hay una diferencia de $0.36 \%$, en el contenido de calcio obtenido utilizando AA e ICP-OES, según los resultados del análisis estadístico, las diferencias entre los tipos de tejido analizados, son altamente significativas, pero el análisis de las técnicas empleadas en cada tejido demuestra que no hay diferencia significativa ( $F$ igual a 0.76 ).

\section{Magnesio}

En la Figura 3 se presentan las concentraciones de magnesio en tejido vegetal de banano, palma y rosas.

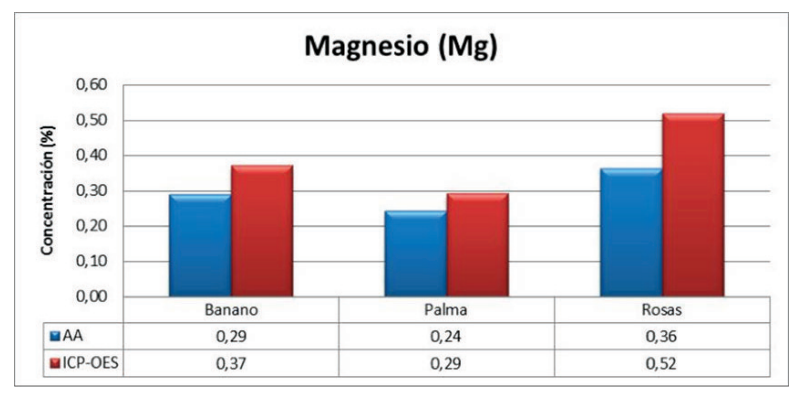

Figura 3. Magnesio en muestras de tejido vegetal

El contenido de magnesio en tejido vegetal de rosas es mayor comparado con los tejidos vegetales de banano y palma analizados, estadísticamente, la diferencia es altamente significativa; al comparar los resultados obtenidos con las técnicas de análisis empleadas, en todas las muestras el contenido del analito es mayor cuando se emplea espectrofotometría de emisión, al aplicar el análisis de varianza se determinó que, estadísticamente, existen diferencias alta- mente significativas entre las técnicas de análisis empleadas para determinar magnesio en los tejidos vegetales del presente estudio.

\section{Zinc}

En la Figura 4 se presentan las concentraciones de zinc en tejido vegetal de banano, palma y rosas, analizados por AA e ICP-OES. 


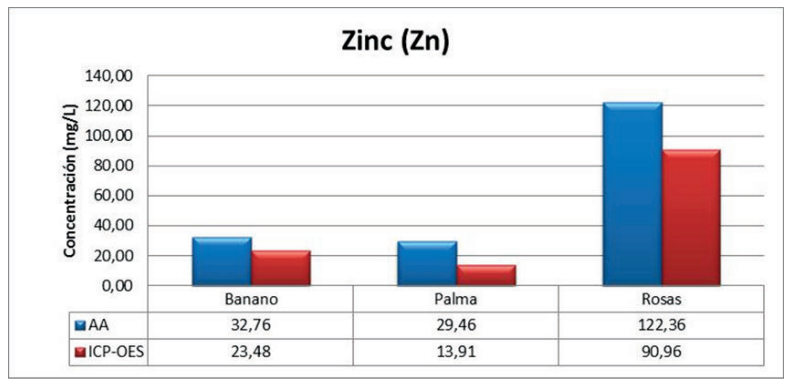

Figura 4. Zinc en muestras de tejido vegetal

El tejido vegetal de rosas contiene mayor cantidad de zinc comparado con el de palma y banano, estadísticamente la diferencia es altamente significativa. El contenido de zinc mediante AA es más alto, en todas las muestras analizadas, y en el caso de tejido vegetal de rosas el contenido es de $122,36 \mathrm{mg} / \mathrm{L}$, y el obtenido por ICP-OES, es de 90,96 mg/L, estadís- ticamente la diferencia es altamente significativa (valor F igual a 21,36).

\section{Cobre}

En la Figura 5 se representan las concentraciones de cobre en los tejidos vegetales de banano, palma y rosas, expresados en $\mathrm{mg} / \mathrm{L}$.

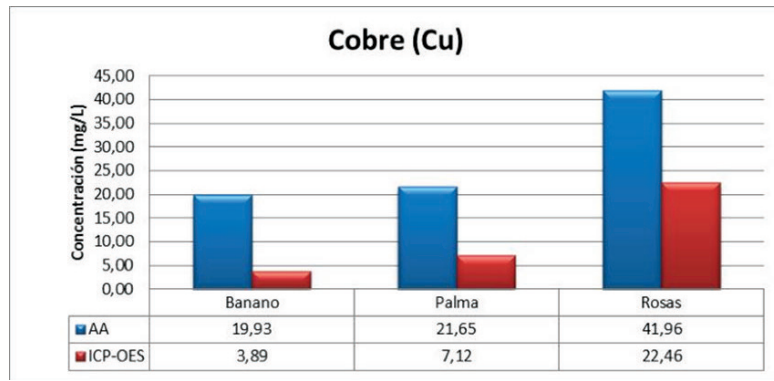

Figura 5. Cobre en muestras de tejido vegetal 
La concentración de cobre determinada por AA e ICP, en las muestras de tejido vegetal de rosas es mayor comparada con los valores obtenidos en las muestras de banano y palma. Los valores obtenidos mediante $A A$, en todos los tejidos analizados, son más altos que los obtenidos por ICP-OES y al comparar los valores obtenidos en un mismo tejido vegetal por las dos técnicas se observa una diferencia que va desde 1,86 a 5,24 veces más alta en AA que en ICP-OES, como sucede en las muestras de banano, por lo tanto hay una diferencia significativa entre las técnicas empleadas.

\section{Hierro}

La Figura 6, corresponde a las concentraciones de hierro en los tejidos vegetales analizados.

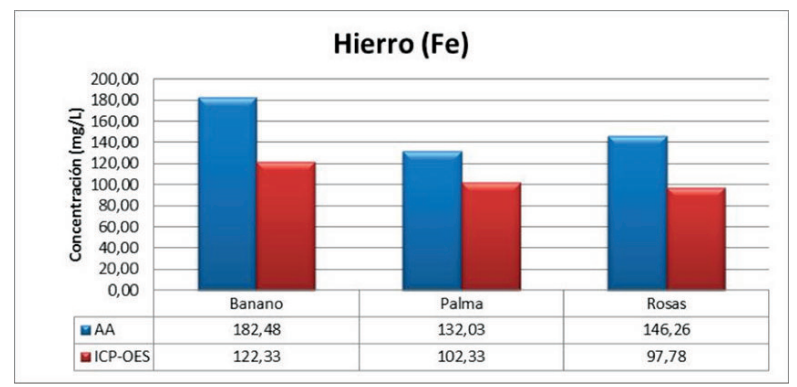

Figura 6. Hierro en muestras de tejido vegetal

Luego de determinar la concentración de hierro en las muestras de tejido foliar, se determinó que existen diferencias altamente significativas entre las técnicas utilizadas en el presente estudio, los valores obtenidos por AA son más altos que los obtenidos por ICP-OES.

\section{Manganeso}

En la Figura 7 se presentan las concentraciones de manganeso en los tejidos vegetales de banano, palma y rosas, analizados por AA e ICP-OES. 


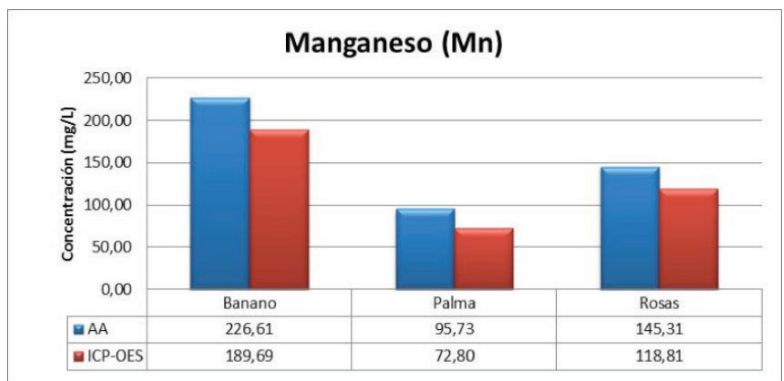

Figura 7. Manganeso en muestras de tejido vegetal

Mediante análisis estadístico se determinó que hay diferencias altamente significativas entre las técnicas utiliza- das para la determinación de manganeso en tejido vegetal y mayor concentración de manganeso se obtiene por AA.

\section{DISCUSIÓN}

Los resultados de este trabajo de investigación demuestran que el potasio es el macronutriente presente en mayor cantidad en las muestras de tejido foliar de banano, seguido del calcio y magnesio, Castillo et.al. (2011) obtuvieron resultados similares al extraer macronutrientes en banano Dominico, el orden de acumulación en el seudotallo del banano fue: potasio, calcio, nitrógeno y magnesio; es de esperar por lo tanto, que el tejido del banano extrae del suelo la mayor cantidad de potasio que el de la palma y las rosas y que este elemento se debe reponer mediante fertilización del suelo de cultivo.
La valores obtenidos al analizar potasio en las muestras de tejido foliar de banano, no presentan una diferencia significativa, por lo tanto, la técnica ICP-OES, puede ser una alternativa para la determinación de este analito en tejido de banano, palma y rosas. Sadzawka et al. (2007), también ha reportado la determinación de fósforo, potasio, calcio y magnesio en tejido vegetal, en un espectrofotómetro de emisión de plasma por inducción acoplada Varian.

En la determinación de los macronutrientes; potasio, calcio y magnesio, mediante las técnicas empleadas en 
este estudio, las concentraciones más altas se obtienen en el análisis de potasio y magnesio mediante ICP-OES, estos valores se justifican debido a las altas temperaturas que alcanza el plasma, por lo cual se eliminan las interferencias químicas que suelen presentarse en absorción atómica.

En el caso de los micronutrientes, a partir del análisis estadístico se deduce que no hay diferencias significativas en las determinaciones de los diferentes tejidos vegetales, mediante las técnicas empleadas en la investigación.

En la digestión ácida se obtuvo una disolución clara, pero no incolora, debido a la permanencia de óxidos de nitrógeno en la disolución. En algunos casos se observó la presencia de sílice como un sólido amorfo. Sin embargo, no presentaron interferencias ya que las muestras fueron atomizadas efectivamente en el ICP debido a la alta temperatura generada por el plasma.

\section{CONCLUSIONES}

Se desarrolló un método analítico que permite utilizar la técnica ICPOES para el análisis multielemental simultáneo de macro y micronutrientes en tejidos foliares de banano, palma y rosas.

Pese a que existió variabilidad en los resultados, las metodologías cumplen de manera general con el criterio establecido, a excepción del análisis de micro-nutrientes en palma, ya que al ser esta una planta oleaginosa, presenta una reacción violenta al momento de la digestión ácida, por lo que se puede perder o contaminar la muestra.
Al realizar el análisis de varianza de las concentraciones de macro y micronutrientes en tejidos vegetales de banano, palma y rosas, se concluyó que existen diferencias estadísticas entre las técnicas de AA e ICP-OES, por lo cual se debería realizar un estudio para determinar cuál técnica es la más adecuada para el análisis foliar.

La lectura de micronutrientes se realizó con el plasma en posición axial, ya que permite obtener mayor sensibilidad y una mejor detección de los analitos a bajas temperaturas. 


\section{BIBLIOGRAFÍA}

Alvarado, A. L., Yglesias, J. M., \& Güel, O. (2005). Análisis Multielemental de Material Foliar por medio de ICP-MS. Obtenido de http://www.mag.go.cr/rev_agr/v29n01 _017.pdf

Boss, C. B., \& Fredeen, K. J. (1997). Concepts, Instrumentation, and Techniques in Inductively Coupled Plasma Optical Emission Spectrometry. U.S.A: The Perkin Elmer Corporation.

Briceño, J., \& Pacheco, R. (1984). Métodos Analíticos para el Estudio de Suelo y Plantas. San José, Costa Rica: Editorial de la Universidad de Costa Rica.

Castillo, A., Hernández, J., Avitia, E., Pineda, J., Valdez, L., Corona, T., (2011). Extracción de macronutrientes en banano "Dominico" (Musa spp). Revista Internacional de Botánica Experimental. 80 65-72.

Instituto Colombiano Agropecuario. (s.f.). El análisis de suelos, plantas y aguas para riego. Manual de Asistencia Técnica № 47.

Sadzawka, R., Carrasco, M., Demanet, R., Flores, H., Grez, R., Mora, M., Neaman, A., (2007). Métodos de análisis de vegetales. $2^{\circ}$ ed. Instituto de Investigaciones Agropecuarias. Chile.

Ulrich, A. (1972). Plant Tissue Analysis. Analysis as guide in fertilizing crops. En Soil and plant tissue testing in California (págs. 18-79).

Westerman, R. L. (1990). Soil tensting. Madison, USA: SSSA. 